Raed Qarajeh, MD

Division of Internal Medicine,

University of Missouri-Kansas City,

Kansas City, MO
Laith A. Derbas, MD

Department of Cardiology,

Rush University Medical Center

Chicago, IL
Mouin Abdallah, MD, MSc Faraz Kureshi, MD, MSc MedStar Washington Hospital Center, St. David's Healthcare, Austin, TX

\title{
A 21-year-old woman with Ehlers-Danlos syndrome and persistent lightheadedness
}

\begin{abstract}
A 2 I-YEAR-OLD WOMAN presents with positional lightheadedness when going from supine to sitting or standing, which is associated with prominent heart palpitations without any overt syncope. She has experienced the symptoms for several years, but the frequency has progressively increased from a few times a month to many times a day. Also, she has a history of unexplained gastrointestinal symptoms including bloating, nausea, and cramping.

Her medical history includes diagnoses of hypermobile (type 3) Ehlers-Danlos syndrome and autoimmune-mediated hypothyroidism, for which she takes oral levothyroxine daily. She had been smoking 1 pack of cigarettes per day for the past 3 years but stopped 2 months ago. She denies using alcohol or recreational drugs.
\end{abstract}

\section{PHYSICAL EXAMINATION}

Sitting, her blood pressure is $106 / 66 \mathrm{~mm} \mathrm{Hg}$ and her pulse is 87 beats per minute and regular. Three minutes after standing, these values are 91/69 $\mathrm{mm} \mathrm{Hg}$ and 115 beats per minute. Her respiratory rate is 16 breaths per minute, and her oral temperature is $36.8^{\circ} \mathrm{C}\left(98.3^{\circ} \mathrm{F}\right)$.

She has no thyromegaly or carotid bruits. On auscultation, her lungs are clear and heart sounds are normal without any murmur. Her abdomen is soft and nontender to palpation. Her extremities are warm, without edema, and have equal, palpable peripheral pulses. The neurologic examination is normal. The musculoskeletal examination is significant for doi:10.3949/ccjm.88a.19137 global hypermobility in her joints.

\section{PREVIOUS TEST RESULTS}

An electrocardiogram a few months before her visit showed normal sinus rhythm, and a transthoracic echocardiogram showed normal cardiac chamber dimensions and biventricular systolic function, a normal mitral valve with trivial regurgitation, and normal ascending aorta dimensions. A 30-day cardiovascular event monitor recorded numerous symptomatic episodes of palpitations and lightheadedness, all of which were associated with sinus tachycardia of varying rates. Her thyroidstimulating hormone level is $1.91 \mathrm{mIU} / \mathrm{L}$ (reference range $0.47-4.68 \mathrm{mIU} / \mathrm{L})$.

\section{WHAT'S CAUSING HER LIGHTHEADEDNESS?}

1

Which of the following is the most likely cause of this patient's symptoms?

Postural orthostatic tachycardia syndrome (POTS)

Orthostatic hypotension

Inappropriate sinus tachycardia

Atrial or ventricular arrhythmia

Our patient has nonspecific symptoms, so the differential diagnosis is broad and could include any of those disorders.

\section{Postural orthostatic tachycardia syndrome}

POTS is commonly characterized by an exaggerated heart rate response without significant orthostatic hypotension in the absence of re-
For several years, a 21 -year-old woman has had lightheadedness upon standing, and it is getting worse 
versible causes such as medications, anemia, or thyroid dysfunction. ${ }^{1}$ It occurs predominantly in women of childbearing age (ages 15-50).

Presenting symptoms are usually nonspecific, such as lightheadedness, blurred vision, palpitations, headache, and nausea. ${ }^{2}$ The syndrome can be associated with several other diseases, including hypermobile Ehlers-Danlos syndrome, an inherited collagen disorder characterized by joint hypermobility. Because of this association, any patient with hypermobile Ehlers-Danlos syndrome and with nonspecific symptoms should be assessed for POTS.

Studies suggest that there is a higher prevalence of POTS in patients with hypermobile Ehlers-Danlos syndrome and that patients with POTS start to experience symptoms at an earlier age if they have a hypermobility syndrome. ${ }^{3-6}$ Rowe et $\mathrm{al}^{3}$ were the first to report an association between POTS and a joint hypermobility syndrome, in 1999. Wallman et $\mathrm{al}^{4}$ found that the hypermobile Ehlers-Danlos syndrome is more prevalent in patients with POTS (18\%) than in the general population $(0.02 \%)$. Gazit et $\mathrm{al}^{5}$ reported that 21 of 27 patients with the syndrome had abnormal autonomic manifestations, such as POTS, orthostatic hypotension,

POTS symptoms are usually nonspecific: lightheadedness, blurred vision, palpitations, headache, and nausea or uncategorized orthostatic intolerance. Kanjwal et $\mathrm{al}^{6}$ found that POTS tends to present at an earlier age in patients with a hypermobile syndrome.

The difference between patients with POTS and hypermobile Ehlers-Danlos syndrome vs those with POTS related to other causes is unclear. However, patients with the syndrome use more healthcare resources, including practitioner evaluations for chronic pain and medications, than those without the syndrome.?

This patient's young age, symptoms, and diagnosis of hypermobile Ehlers-Danlos syndrome raise suspicion of POTS and make it the most likely diagnosis. However, POTS can be self-limited or follow a relapsing-remitting pattern over several years, which can make it difficult to diagnose.

\section{Orthostatic hypotension}

The classic orthostatic hypotension symptom is a significant drop in blood pressure when standing up from sitting or lying down. The consensus definition is a fall in systolic blood pressure of at least $20 \mathrm{~mm} \mathrm{Hg}$ or diastolic blood pressure of at least $10 \mathrm{~mm} \mathrm{Hg}$ within 3 minutes of standing. Orthostatic hypotension can be symptomatic or asymptomatic. It has a higher prevalence in patients over age 70 and is usually associated with other disorders such as diabetes mellitus, Parkinson disease, pure autonomic failure, and multiple system atrophy. ${ }^{8-10}$

This patient's lack of orthostatic blood pressure drop, young age, and absence of associated comorbidities makes this diagnosis less likely.

\section{Inappropriate sinus tachycardia}

This syndrome is defined as sinus tachycardia of more than 100 beats per minute at rest with a mean 24-hour heart rate of more than 90 beats per minute that is not due to primary causes. It is often associated with distressing palpitation symptoms.

Inappropriate sinus tachycardia is induced by both physiologic and emotional stresses; however, POTS is induced only by orthostasis. Use of a 24-Holter monitor can confirm inappropriate sinus tachycardia.

Although there may be an overlap between POTS and inappropriate sinus tachycardia, the latter is not likely in this patient because her resting heart rate was not more than 100 beats per minute, a diagnostic requirement of the syndrome.

\section{Atrial or ventricular arrhythmia}

Atrial or ventricular arrhythmias can cause syncope or near-syncope. These are broad categories that can include ventricular tachycardia, sinoatrial node dysfunction, atrioventricular node block, and atrial arrhythmias such as supraventricular tachycardia, fibrillation, or flutter.

This patient had normal sinus rhythm, and her 30-day event monitor showed no arrhythmias, making an atrial or ventricular cardiac arrhythmia unlikely.

\section{WHAT ABOUT HER EHLERS-DANLOS SYNDROME?}

2 What is the inheritance pattern of hypermobile Ehlers-Danlos syndrome?

Autosomal dominant

Autosomal recessive

X-linked dominant 


\section{Autosomal dominant}

Although the gene or genes responsible for this syndrome have not been identified, it appears to follow an autosomal dominant pattern of inheritance. ${ }^{11}$

\section{Autosomal recessive}

Other clinical subtypes of the Ehlers-Danlos syndrome, including classic, cardiac-valvular, dermatosparaxis, kyphoscoliotic, spondylodysplastic, and musculocontractural, follow an autosomal recessive pattern of inheritance; however, the hypermobile Ehlers-Danlos syndrome subtype does not. ${ }^{12}$

\section{X-linked dominant}

None of the clinical subtypes follow an Xlinked dominant inheritance in the $2017 \mathrm{di}$ agnostic criteria. The X-linked Ehlers-Danlos syndrome with muscle hematoma is no longer included in the syndrome spectrum. ${ }^{12}$

\section{BACKGROUND ON EHLERS-DANLOS SYNDROME CLASSIFICATIONS}

Hypermobile is only 1 category of Ehlers-Danlos syndrome, albeit the most common. In the late 1960s, the Ehlers-Danlos syndrome was classified into 5 distinct subtypes. ${ }^{13,14}$ In 1986, an international workshop held in Berlin on classification (nosology) of inherited connective tissue diseases identified 11 subtypes of Ehlers-Danlos syndrome based on the inheritance patterns and phenotypic presentation. ${ }^{15}$ A significant portion of their diagnostic criteria was based on subjective assessment and, thus, is not very reliable.

Improved understanding of the molecular basis for Ehlers-Danlos syndrome led to the 1997 Villefranche revised nosology, which classified the syndrome into 6 subtypes. ${ }^{16}$ The major diagnostic criteria for the hypermobile Ehlers-Danlos syndrome subtype were generalized joint hypermobility and skin involvement. Minor diagnostic criteria included recurring joint dislocations, chronic joint or limb pain, and a positive family history. Joint hypermobility should be assessed using the Beighton scoring system, with hypermobility defined as a score of 5 of 9 or greater. ${ }^{16}$

\section{Date of her diagnosis important}

This patient was evaluated by a rheumatologist in 2016, found to have a 7 of 9 Beigh- ton score, and diagnosed with hypermobile Ehlers-Danlos syndrome based on the Villefranche nosology criteria. In 2017, the International Consortium published new diagnostic criteria for Ehlers-Danlos syndrome that identified 13 unique subtypes. ${ }^{12}$ These criteria include extra-articular features and more strict diagnostic criteria for the hypermobile Ehlers-Danlos syndrome.

In a recent study by Miller et $\mathrm{al}^{17}$ that looked at the prevalence of hypermobile Ehlers-Danlos syndrome in patients with POTS, only 19 $(56 \%)$ of 32 patients who self-reported a hypermobile Ehlers-Danlos syndrome diagnosis met the 2017 diagnostic criteria. Therefore, it is always important to confirm a self-reported diagnosis using the new criteria.

We know that this patient was diagnosed according to the Villefranche criteria, but we don't know whether the diagnosis was updated using the 2017 criteria. That may not matter, because the new criteria have been criticized for being too restrictive. McGillis et al ${ }^{18}$ reported that the 2017 diagnostic criteria leave many highly symptomatic patients without a diagnosis. Further, the newest diagnostic criteria have not been validated and, thus, require refinement to improve their diagnostic accuracy.

\section{CASE CONTINUED: DIAGNOSING POTS}

Comorbidities usually associated with hypermobile Ehlers-Danlos syndrome include chronic pain, gastrointestinal dysfunction, ${ }^{19}$ orthostatic intolerance, and POTS. ${ }^{20}$ Voermans et $\mathrm{al}^{21}$ reported that $29(73 \%)$ of 40 patients with Ehlers-Danlos syndrome had myalgia continuously or frequently after exercise.

Our patient has gastrointestinal symptoms including bloating, nausea, and cramping. She also has chronic generalized pain that has required multiple emergency department visits. At this point, POTS is still the most likely diagnosis of her lightheadedness.

Which of the following is the most appropriate test to diagnose POTS?

Tilt-table testing

24-hour ambulatory blood pressure recording

Plasma norepinephrine measurement
Comorbidities associated with hypermobile Ehlers-Danlos syndrome: chronic pain, gastrointestinal dysfunction, orthostatic intolerance, and POTS 


\section{Tilt-table testing}

The correct answer is tilt-table testing. The diagnostic criteria for POTS include at least 3 months of symptoms that increase on standing and improve when lying down, plus either a heart rate increase of more than 30 beats per minute or a sustained heart rate of at least 120 beats per minute, and a nonsignificant drop in blood pressure $(<20 / 10 \mathrm{~mm} \mathrm{Hg}) .{ }^{22}$ Children and adolescents can have a slightly higher physiologic range and require a heart rate increase of more than 40 beats per minute for the diagnosis. ${ }^{1}$ Tilt-table testing is the definitive test, as it is done in a controlled setting with few variables that may alter the accuracy of results. ${ }^{23}$

\section{4-hour ambulatory blood pressure recording}

A 24-hour ambulatory blood pressure recording is not the correct test, as it would not be helpful in the evaluation of POTS. In order to diagnose POTS, we need a diagnostic test that evaluates changes in heart rate and blood pressure in both the supine and upright positions in a controlled setting-like the tilt-table test.

\section{Plasma norepinephrine measurement}

She experienced A hyperadrenergic variant form of POTS has gradual improvement in her symptoms and quality of life been defined as an increase in plasma norepinephrine levels at rest and on standing (serum norepinephrine level $\geq 600 \mathrm{pg} / \mathrm{mL}$ ). This would not be the initial test for evaluating patients with suspected POTS because the sensitivity and specificity of this test are not clear for POTS.

\section{Back to the patient}

Our patient underwent tilt-table testing. Her baseline heart rate was 84 beats per minute in sinus rhythm and her blood pressure was $114 / 74 \mathrm{~mm} \mathrm{Hg}$. She was then moved to a $70 \%$ tilt position. Immediate upright vital signs were heart rate 113 beats per minute and blood pressure 105/77 $\mathrm{mm} \mathrm{Hg}$. She was kept in the head-up tilt position for a total of 30 minutes. During this time, vital signs were monitored every 1 minute. The patient reported symptoms of lightheadedness, extreme fatigue, difficulty catching breath, and weakness during this 30-minute period. Her heart rate ranged from a low of 106 beats per minute and a high of 152 beats per minute. It increased to 120 beats per minute at 3 minutes upright and stayed 120 to 150 beats per minute for the remainder of upright positioning. She had no significant fall in blood pressure during tilting. After resuming the supine position, the patient had immediate recovery of heart rate at 86 beats per minute with blood pressure of 109/64 mm Hg. In addition, all her symptoms resolved. Therefore, our patient had a positive tilt-table test for POTS.

\section{MANAGING POTS}

4

Which of the following is the most appropriate initial step in managing POTS for this patient?

Nonpharmacologic treatment and lifestyle modifications

Start a beta-blocker

Start midodrine

Start pyridostigmine

\section{Nonpharmacologic treatment and lifestyle modifications}

Nonpharmacologic treatment should be used first. ${ }^{1}$ Management should aim at withdrawing medications that might worsen POTS, avoiding dehydration, increasing fluid intake (up to $2 \mathrm{~L}$ per day), increasing daily salt intake (up to 3-5 g), wearing waist-high compression stockings to decrease venous pooling, and engaging in aerobic and some resistance exercises involving the legs and abdomen. ${ }^{1,6}$

\section{Pharmacologic therapy}

Usually, multiple approaches are needed to treat patients with POTS. Several medications are used off-label, including fludrocortisone, beta-blockers, midodrine, clonidine, methyldopa, and pyridostigmine. The choice of therapy should be based on clinical expertise and patient tolerance or response.

We counseled our patient about lifestyle modifications and gradually started her on fludrocortisone, a beta-blocker in a low dose, and pyridostigmine. She experienced gradual improvement in her symptoms and quality of life.

\section{DISCLOSURES}

The authors report no relevant financial relationships which, in the context of their contributions, could be perceived as a potential conflict of interest. 


\section{REFERENCES}

1. Sheldon RS, Grubb BP 2nd, Olshansky B, et al. 2015 Heart Rhythm Society expert consensus statement on the diagnosis and treatment of postural tachycardia syndrome, inappropriate sinus tachycardia, and vasovagal syncope. Heart Rhythm 2015; 12(6):e41-e63. doi:10.1016/j.hrthm.2015.03.029

2. Jacob G, Costa F, Shannon JR, et al. The neuropathic postural tachycardia syndrome. N Engl J Med 2000; 343(14):1008-1014. doi:10.1056/NEJM200010053431404

3. Rowe PC, Barron DF, Calkins H, Maumenee IH, Tong PY, Geraghty MT. Orthostatic intolerance and chronic fatigue syndrome associated with Ehlers-Danlos syndrome. J Pediatr 1999; 135(4):494-499. doi:10.1016/s0022-3476(99)70173-3

4. Wallman D, Weinberg J, Hohler AD. Ehlers-Danlos syndrome and postural tachycardia syndrome: a relationship study. J Neurol Sci 2014; 340(1-2):99-102. doi:10.1016/j.jns.2014.03.002

5. Gazit Y, Nahir AM, Grahame R, Jacob G. Dysautonomia in the joint hypermobility syndrome. Am J Med 2003; 115(1):33-40. doi:10.1016/s0002-9343(03)00235-3

6. Kanjwal K, Saeed B, Karabin B, Kanjwal Y, Grubb BP. Comparative clinical profile of postural orthostatic tachycardia patients with and without joint hypermobility syndrome. Indian Pacing Electrophysiol J 2010; 10(4):173-178. pmid:20376184

7. Miglis MG, Schultz B, Muppidi S. Postural tachycardia in hypermobile Ehlers-Danlos syndrome: a distinct subtype? Auton Neurosci 2017; 208:146-149. doi:10.1016/j.autneu.2017.10.001

8. Low PA. Prevalence of orthostatic hypotension. Clin Auton Res 2008; 18(suppl 1):8-13. doi:10.1007/s10286-007-1001-3

9. Low PA, Denq JC, Opfer-Gehrking TL, Dyck PJ, O'Brien PC, Slezak JM. Effect of age and gender on sudomotor and cardiovagal function and blood pressure response to tilt in normal subjects. Muscle Nerve 1997; 20(12):1561-1568. doi:10.1002/(sici)1097-4598(199712)20:12<1561::aid-us11>3.0.co;2-3

10. Allcock LM, Ullyart K, Kenny RA, Burn DJ. Frequency of orthostatic hypotension in a community based cohort of patients with Parkinson's disease. J Neurol Neurosurg Psychiatry 2004; 75(10):1470-1471. doi:10.1136/jnnp.2003.029413

11. Tinkle B, Castori M, Berglund B, et al. Hypermobile Ehlers-Danlos syndrome (a.k.a. Ehlers-Danlos syndrome type III and Ehlers-Danlos syndrome hypermobility type): clinical description and natural history. Am J Med Genet C Semin Med Genet 2017; 175(1):48-69. doi:10.1002/ajmg.c.31538

12. Malfait F, Francomano C, Byers P, et al. The 2017 international classification of the Ehlers-Danlos syndromes. Am J Med Genet C Semin
Med Genet 2017; 175(1):8-26. doi:10.1002/ajmg.c.31552

13. Barabas AP. Heterogeneity of the Ehlers-Danlos syndrome: description of three clinical types and a hypothesis to explain the basic defect(s). Br Med J 1967; 2(5552):612-613. doi:10.1136/bmj.2.5552.612

14. Beighton P. Ehlers-Danlos syndrome. Ann Rheum Dis 1970; 29(3):332-333. doi:10.1136/ard.29.3.332

15. Beighton P, de Paepe A, Danks D, et al. International nosology of heritable disorders of connective tissue, Berlin, 1986. Am J Med Genet 1988; 29(3):581-594. doi:10.1002/ajmg.1320290316

16. Beighton P, De Paepe A, Steinmann B, Tsipouras P, Wenstrup RJ. Ehlers-Danlos syndromes: revised nosology, Villefranche, 1997 Ehlers-Danlos National Foundation (USA) and Ehlers-Danlos Support Group (UK). Am J Med Genet 1998; 77(1):31-37. doi:10.1002/(sici)1096-8628(19980428)77:1<31::aid-ajmg8>3.0.co;2-o

17. Miller AJ, Stiles LE, Sheehan T, et al. Prevalence of hypermobile Ehlers-Danlos syndrome in postural orthostatic tachycardia syndrome. Auton Neurosci 2020; 224:102637. doi:10.1016/j.autneu.2020.102637

18. McGillis L, Mittal N, Santa Mina D, et al. Utilization of the 2017 diagnostic criteria for hEDS by the Toronto GoodHope Ehlers-Danlos syndrome clinic: a retrospective review. Am J Med Genet A 2020; 182(3):484-492. doi:10.1002/ajmg.a.61459

19. Fikree A, Chelimsky G, Collins H, Kovacic K, Aziz Q. Gastrointestinal involvement in the Ehlers-Danlos syndromes. Am J Med Genet C Semin Med Genet 2017; 175(1):181-187. doi:10.1002/ajmg.c.31546

20. Hakim A, O'Callaghan C, De Wandele I, Stiles L, Pocinki A, Rowe P. Cardiovascular autonomic dysfunction in Ehlers-Danlos syndromehypermobile type. Am J Med Genet C Semin Med Genet 2017; 175(1):168-174. doi:10.1002/ajmg.c.31543

21. Voermans NC, van Alfen N, Pillen S, et al. Neuromuscular involvement in various types of Ehlers-Danlos syndrome. Ann Neurol 2009; 65(6):687-697. doi:10.1002/ana.21643

22. Grubb BP, Kanjwal Y, Kosinski DJ. The postural tachycardia syndrome: a concise guide to diagnosis and management. J Cardiovasc Electrophysiol 2006; 17(1):108-112. doi:10.1111/j.1540-8167.2005.00318.x

23. Plash WB, Diedrich A, Biaggioni I, et al. Diagnosing postural tachycardia syndrome: comparison of tilt testing compared with standing haemodynamics. Clin Sci (Lond) 2013; 124(2):109-114. doi:10.1042/CS20120276

Address: Raed Qarajeh, MD, Division of Internal Medicine, University of Missouri-Kansas City, 2301 Holmes Street, Kansas City, MO 64108; qarajehr@umkc.edu 